\title{
Optical Coherence Tomography Angiography Study for Evaluation of Microvascular Changes in the Subclinical Diabetic Retinopathy
}

\author{
Hisham Fawzy Khalil, Ahmed Elsayed Hodaib, Ahmed Younis Elsaeid \\ Department of Ophthalmology, Faculty of Medicine, Al-Azhar University
}

Corresponding author:Ahmed YounisElsaeid, Mobile: 00201110531368, 01005025004 E-Mail:ahmedyounes143@yahoo.com, ahmedyounis@ebsareye.com

\begin{abstract}
Background: diabetic retinopathy is the specific microvascular complication of diabetes mellitus and affects 1 of 3 with DM. DR remains a leading cause of vision loss in working adult population. Patients with severe levels of DR were reported to have poor quality of life and reduced physical, emotional and social well-being, and they utilize more health care resources. Objective: this study aimed to evaluate the vascular density, perfusion density and foveal avascular parameters changes in subjects with diabetes mellitus showing no manifestations of diabetic retinopathy using optical coherence tomography angiography. Methods: 40 subjects were categorized into two groups, group A for healthy subjects and group B for the diabetic subjects. HbA1c, renal functions, liver functions and lipid profile were obtained from each individual. Best corrected visual acuity were obtained from each individual and each were subjected to full ophthalmic examination, IOP measurement, fundus fluorescein angiography FFA, Optical Coherence Tomography OCT and Optical Coherence Tomography Angiography OCTA. Results: statisticallysignificantdifferenceswerefoundbetweenthehealthygroupand the diabeticgroupin OCTA parameters. Full vascular density VD significantly decreases in the diabetic patients even in those showed BCVA $\geq 0.7$ and totally intact FFA and OCT $(\mathrm{P}>0.001)$. Perfusion density PD also was significantly decreased in the diabetic patients $(\mathrm{P}>0.001)$. Foveal avascular zone showed significant changes in area value and perimeter, however the circularity changes were statisticallyinsignificant.Conclusion: using OCTA was proven to be clinically significant in differentiating healthy eyes from diabetic eyes that showed no clinically acceptable hallmarks. So, that the term "Subclinical diabetic retinopathy" was suggestible to refer to a clinical state where the FAZ metrics, vascular density and perfusion density showed a particular deviation from the normal healthy parameters.
\end{abstract}

Keywords -OpticalCoherence Tomography Angiography, Diabetic Retinopathy

\section{Introduction}

Diabetic retinopathy is the specific microvascular complication of diabetes mellitus and affects 1 of 3 with DM. DR remains a leading cause of vision loss in working adult population. Patients with severe levels of DR are reported to have poor quality of life and reduced physical, emotional and social well-being, and they utilize more health care resources ${ }^{[1]}$. Recent studies ${ }^{[2-}$ ${ }^{5]}$ supported the concept that the retinal vasculature may provide a summary measure of lifetime exposure to the effects of hyperglycemia. Advances in retinal photographic techniques and in image analysis allowed objective and precise in vivo measurement of retinal vascular changes. In particular quantitative assessment of retinopathy signs and measurement of retinal vascular caliber have greatly increased our knowledge of early microcirculation alterations in prediabetes, diabetes and diabetic macro and microcirculation complication ${ }^{[6]}$.Clinically, DR has two principal stages.The first phase, nonproliferative DR (NPDR), is characterized by retinal microvascular changes such as pericyte loss, basement membrane thickening, formation of microaneurysms and intraretinalmicrovascular abnormalities. The more serious proliferative DR (PDR) stage is characterized by the development of new blood vessels (neovascularisation) NV's on the surface of the retina. This in turn leads to fibrovascular changes and tractional retinal detachment resulting in blindness ${ }^{[6]}$. Fluorescein angiography or color fundus photographs have 
been used to establish quantitative indices of perfusion in $\mathrm{DR}^{[7]}$. However, these imaging modalities do not resolve retinal capillaries reliably $^{[8]}$ and cannot detect subtle changes. Optical coherence tomography (OCT) became a part of the standard of care in ophthalmology. It provided cross-sectional and three dimensional imaging of the anterior segment, retina and optic nerve head with micrometer scale-depth resolution, Structural OCT enhances the clinician's ability to detect and monitor fluid exudation associated with vascular diseases ${ }^{[9]}$. Itis however unable to directly detect capillary dropout or pathological vessel growth( neovascularizarion) that constitutes the major vascular changes associated with two leading causes of blindness, age-related macular degeneration "AMD" and diabetic retinopathy, These features among other vascular abnormalities are assessed clinically using fluorescein or indocyanine green angiography (ICG), To overcome conventional structural OCT's inability to provide direct blood flow information, several OCT angiography OCTA methods had been developed ${ }^{[10]}$.Quantification of retinal perfusion using OCTA has been reported in normal subjects ${ }^{[1,12]}$ and retinal vascular disease $^{[13]}$. OCTA provided a novel method for non-invasively imaging capillary network and the foveal avascular zone $e^{[14,15]}$. Also, OCTA can use split-spectrum amplitude-decorrelation angiography algorithm to detect erythrocyte movement ${ }^{[16]}$. The currently commercially available OCTA machines allowed a four-section division of the retina-choroid complex: superficial capillary plexus (SCP), DCP, outer retinal layers and choriocapillaris. A new software update allowed quantification of the vascular density "VD" around the macula. Vascular density was defined as the percentage of the sample area occupied by vessel lumens following binary reconstruction of images. In vivo quantification of $\mathrm{VD}$ and the foveal avascular zone FAZ area may be useful in detecting and monitoring the progression of retinal vascular diseases such as diabetic retinopathy, retinal vein occlusion, and radiation retinopathy ${ }^{[17]}$.

\section{Aim of the Work:}

This study aimed to evaluate the vascular density, perfusion density and foveal avascular parameters changes in subjects with diabetes mellitus showing no manifestations of diabetic retinopathy using optical coherence tomography angiography.

\section{Patients and Methods:}

An observational cross-sectional study was performed to evaluate microvascular changes in the diabetic patients, diagnosed with type II diabetes mellitus from 4 to 8 years, showed no frank manifestations of the diabetic retinopathy neither on clinical examination nor on the common diagnostic tools " Fundus fluorescein angiography and OCT".This study included 80 eyes of 40 subjects. 20 of the subjects were diabetic and the other 20 were normal controls. Group A was the healthy subjects and Group B was the diabetic subjects.

\section{The study was approved by the Ethics Board of Al-Azhar University. \\ Inclusion criteria for group A involved no} history of chronic vascular or metabolic disease, $\mathrm{BCVA} \geq 1.0$ and normal ocular examination whereas the exclusion criteria involved Systemic, vascular or metabolic disease, history of eye surgery " any type of intraocular surgery or refractive surgery", high normal or high IOP and physiological cupping. Inclusion criteria for group B involved patients with the diabetes mellitus $\geq 5$ years and $\leq 8$ years without diabetic retinopathy. Whereas, the exclusion criteria for group $b$ involved the presence of diabetic retinopathy or diabetic macular oedema. presence of disabling media opacities ie; AC flare,significant cataract, vitreous opacities, past intravitreal injection of either Anti- Vascular endothelial growth factor "Anti-VEGF" or steroids, past cataract extraction surgery or vitrectomy or pseudophakia, past focal, grid laser or panretinal photocoagulation PRP, presence of any other vascular or metabolic diseases than DM or presence of hereditary or acquired macular dystrophic disease. All laboratory investigations were done after 12 hours of fasting to evaluatediabetes profile ( HbA1C, FBS), kidney profile (Blood urea, S.Creatinine), liver profile ( S.GPT"ALT”, S, GOT “AST”)andlipid profile ( Cholesterol, triglycerides, HDL-C, LDL-C, TC/HDL ratio, LDL/HDL ratio). Full 
Ophthalmic examination was performed to assess the Uncorrected visual acuity "UCVA" as well as Best corrected visual acuity "BCVA by landot $\mathrm{C}$ optotype using snellen chart. Pupil reaction was assessed, slit lamp biomicroscopy, to assess corneal clarity and integrity, depth of the anterior chamber, dilatation of the pupil, clarity of the crystalline lens, clarity of the vitreous cavity, anatomical and morphological integrity of the optic disc and posterior pole using noncontact digital widefield lens by Volk USAfor indirect ophthalmoloscopy and intraocular pressure by using goldmannapplanation tonometer.

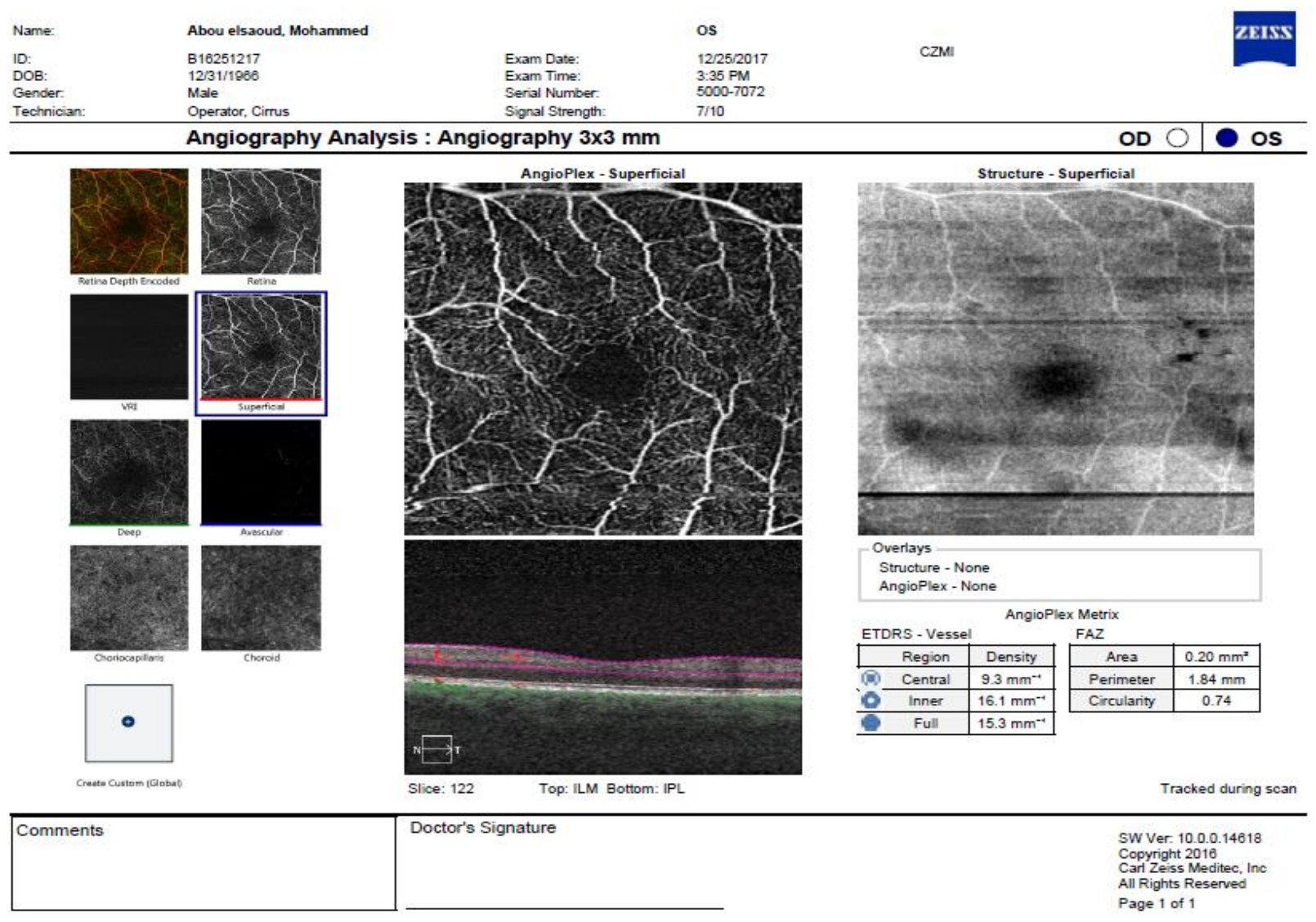

Figure 1- OCTA automated analysis of the vascular density and Foveal avascular zone parameters in a diabetic patient 


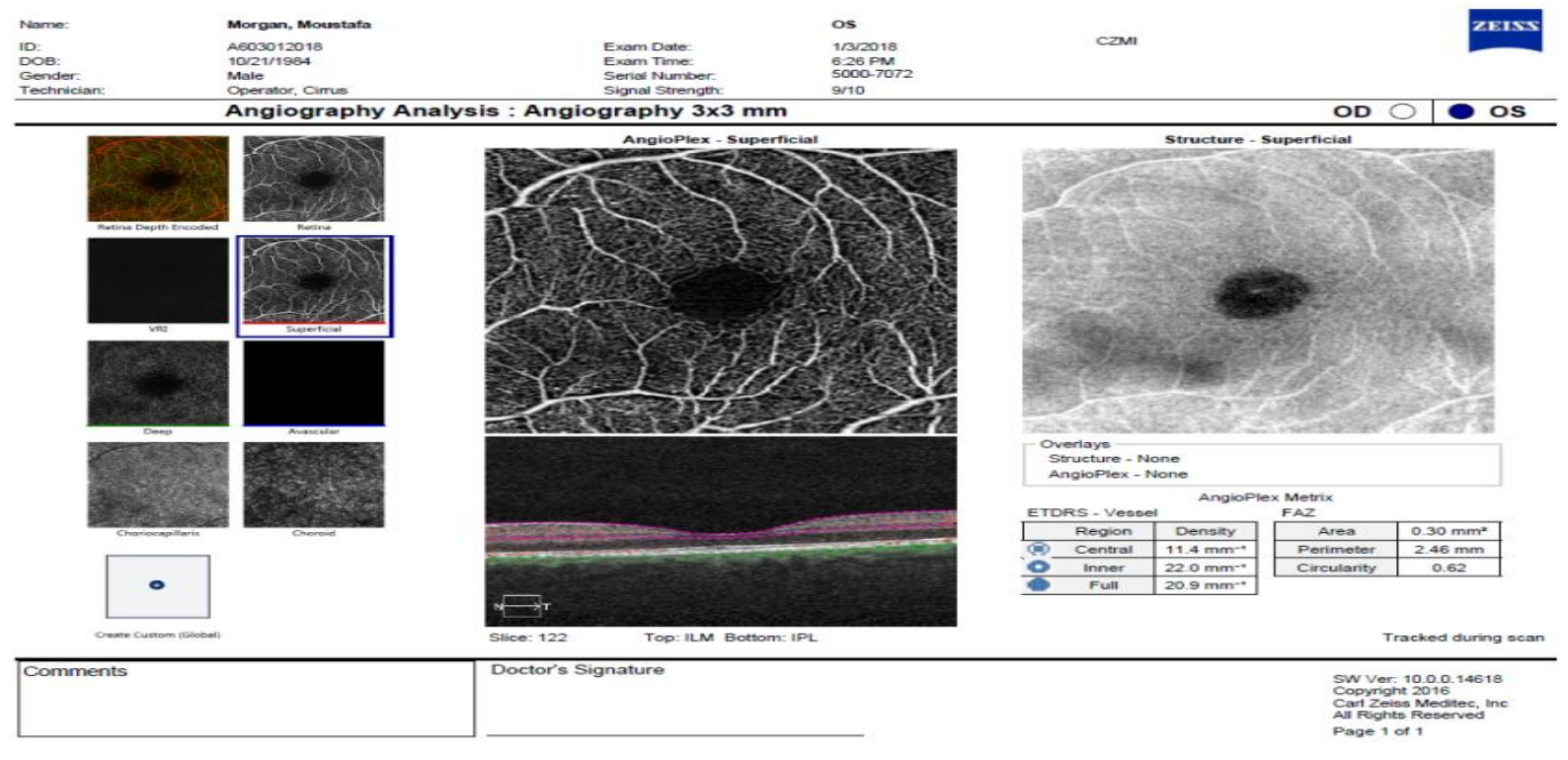

Figure 2- OCTA automated analysis of vascular density and Foveal avasculara zone parameters in a healthy volunteer

Colored fundoscopic picture, fundus fluorescein angiography, OCT and OCT Angio were performed to assess the state of patients' neurosensory retina and retinal vasculature. Optical Coherence tomography OCT retinal map and corss lines were obtained using the highdefinition OCT " Cirrus by Carl ZeissMeditec, Inc". Optical coherence tomographic angiography imaging was performed also with a Cirrus high-definition-OCT prototype AngioPlex instrument using the Optical Micro Angiography algorithm (Carl ZeissMeditec, Inc). Both eyes of each participant were scanned with a scan comprising 245 clusters of B-scans repeated 4 times, where each B-scan consisted of 245 A-scans. The effect of eye motion-related artifacts was minimized by the use of tracking.The averaged OCTB-scans were also examined in the usual manner of OCT data to show the retinal tissue. This volume of data was segmented using the CIRRUS inner-limiting membrane and retinal pigment epithelium segmentation algorithms. From these layers, estimates were derived to subdivide the inner retina into 2 distinct physiologic layers: a superficial retinal layer (SRL) and a deeper retinal layer (DRL).The inner retina was estimated as being the tissue between the innerlimiting membrane and an offset from the retinal pigment epithelium of $110 \mu \mathrm{m}$.The SRL was defined as the inner $70 \%$ of the inner retina, and the DRL was the remaining $30 \%$ of the inner retina.Thelayerestimateswere applied to the 3dimensional Optical Micro Angiography algorithm motion contrast data set. A maximum projection method within the layer of interest was used to generate the en face images. To calculate the perfusion density and vessel density a thresholding algorithm was applied to the SRL or DRLen face images to create a binary slab that assigns to each pixel a 1 (perfused) or 0 (background). From this slab, a skeletonized slab was created, representing vesselswith a trace of 1 pixel in width $^{[18]}$. Using an existing algorithm available on the Cirrus device, we used the fovea position as the starting point for an iterative region-growing algorithm that identified the FAZ. The area and perimeter of this zone were calculated, and the circularity index was calculated as $4 \pi \mathrm{A} / \mathrm{P}$, whereas the area and $\mathrm{P}$ is the perimeter.2 The FAZ measures were based only on the SRL because there is only expected to be a single capillary plexus at the border of the FAZ $^{[18]}$. 


\section{Results:}

Statistical analysis of the outcomes showed that the mean of the vascular density VD in the control group was $20.40 \pm 1.56$ which showed a statistically significant difference from the diabetic group that showed a mean VD of $17.65 \pm 2.87(\mathrm{P}<0.001)$. Perfusion density also appeared to be an important biomarker in the diabetic patients, the mean perfusion density PD in the healthy group was $0.36 \pm 0.06$ while, in the diabetic group it was $0.33 \pm 0.04(\mathrm{P}<0.001)$ showing a statistically significant difference between both groups. Foveal avascular zone FAZ was numerically assessed by measuring the perimeter $\mathrm{P}$, area $\mathrm{A}$ and circularity index $\mathrm{C}$. By comparing these values in both groups FAZ-A showed a statistically significant difference (P 0.017 ) the mean value in the control group was $0.26 \pm 0.07$ while in the diabetic group it was 0.32 \pm 0.12. FAZ-P also showed a statistically significant difference ( $P$ 0.011) where the mean value in the control group was $2.18 \pm 0.33$ and in the diabetic group was $2.46 \pm 0.58$, it seemed that FAZ perimeter increases in the diabetic patients. The only value that didn't show a statistically significant difference in the FAZ-C (P 0.102)with a mean value of $0.67 \pm 0.08$ in the control group and $0.63 \pm 0.12$ in the diabetic group.

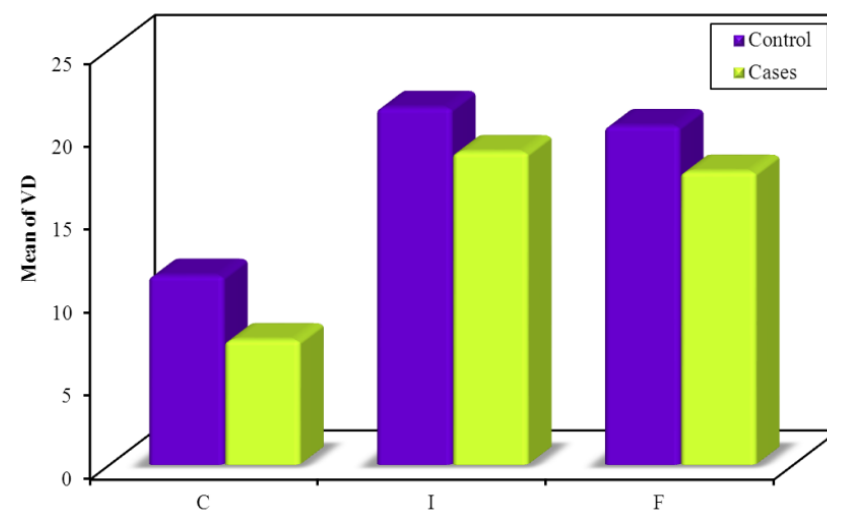

Figure 3 : mean value of vascular density difference between the control group " blue " and the diabetic group "yellow".

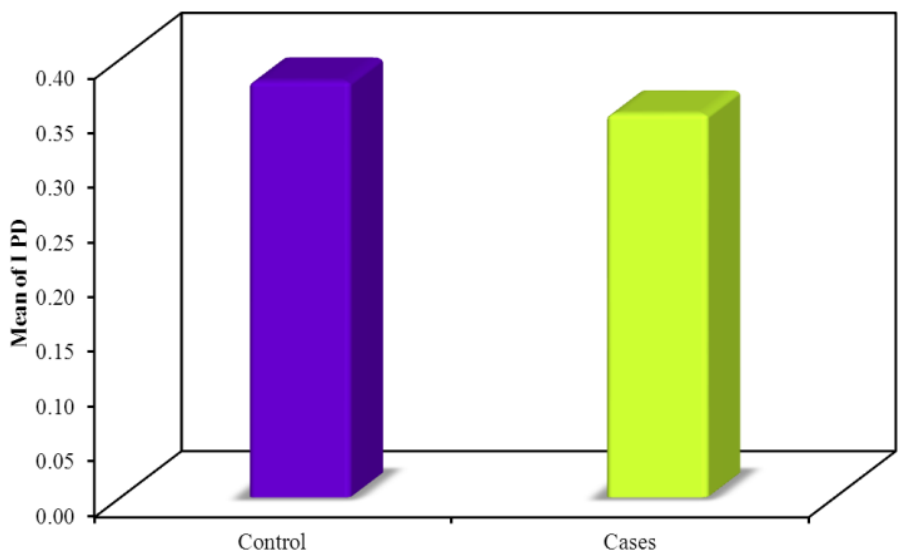

Figure 4 : mean value of perfusion density difference between the control group "blue " and the diabetic group "yellow"

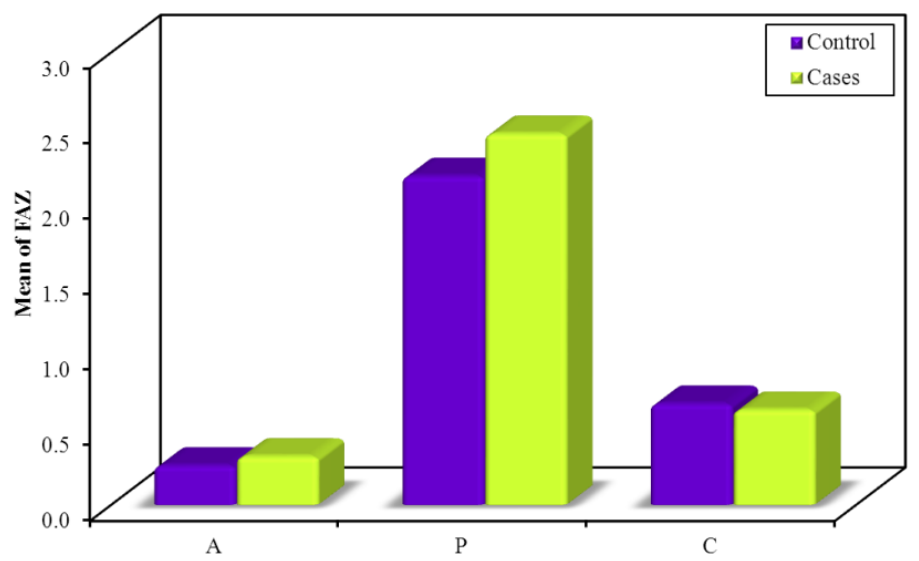

Figure 5 : mean values of the foveal avascular zone difference between the control group " blue " and the diabetic group "yellow"

\section{Discussion:}

laboratory in vitro research work suggested that hyperglycemia and diabetes mellitus influences the normal histological status of the retinal tissue and the vessels themselves, by influencing the perfusion density of the tissue, this was unidentifiable by the normal diagnostic tools "FFA and OCT" since the earliest sign could be identified by FFA with the presence of microaneurysms, which is a relatively late pathological entity ${ }^{[19]}$. Tam et al $^{\left[{ }^{[20]}\right.}$ published a study entitled "Subclinical Capillary Changes in Non-proliferative Diabetic retinopathy" to establish adaptive optics scanning laser ophthalmoscopy (AOSLO) as a method to detect and characterize microscopic signs of diabetic 
retinopathy, in capillaries and cone photoreceptors in the parafovea. The study concluded that "AOSLO imaging can be used to longitudinally track capillaries, leukocytes and photoreceptors in diabetic retinopathy. Capillary changes that can be detected include dropout of individual capillaries, as well as formation and disappearance of microaneurysms".A study published by Christophet al. ${ }^{[21]}$ entitled " Molecular imaging of subclinical diabetic retinopathy". The article explaineda new laboratory method published by Frimmel et $\boldsymbol{a l} .^{[22]}$ as one in a series of articles from Hafezi-Moghadam et al..$^{[23]}$ at Harvard Medical School and the Brigham and Women's Hospital that offer a diametrical departure from existing approaches to early detection of DR. To visualize the earliest vascular changes in diabetes, he developed a novel and nature-inspired molecular imaging approach, as the authors explain in their discussion. Using their custom-designed probes in combination with scanning laser ophthalmoscopy, in their work elegantly visualized early molecular signs of DR. In recruiting volunteers for the study, we made an announcement for individuals with type II diabetes mellitus, we made the onset of the disease clear and required the volunteers to be symptomatically free and have no complain. It was tricky for most of the participants why do we need " asymptomatic subjects" and it wasn't easy to explain to each one the idea of preventive and prophylactic procedures in modern medicine, such thing was considered a luxury in our health care system, while wasn't not so in other areas.All the volunteers got a "patient education session" about diabetes mellitus and proper nutrition, lifestyle and medical follow up to avoid the hazards of systemic diabetic complications. We paid a special attention to diabetic vascular complications and diabetic retinopathy and the way to coexist with it without letting the disease defeats you. We took an advantage of the opportunity to talk over modern methods for intervention and treatment of complications of diabetic retinopathy, since all the participants are expected to develop diabetic retinopathy complications sooner or later. It's known that nearly all the patients showed some kinds of diabetic retinopathy after two decades of the onset of the disease. Three of the volunteers got diagnosed with diabetic retinopathy and two of them with cataract, so we excluded them from the study and directed them to the proper treatment. Two of the participants were diagnosed with unilateral amblyopia, so we excluded only the amblyopic eye and involved the normal eye in the study. Only one participant refused the fluorescein angiography test, so he got excluded from the study. Device selection: this clinical study was done by using the AngioPlex OCTA from Zeiss Meditec, Germany. During the study, we had accessibility to other commercially available devices like the Topcon SS Triton++ OCTA and AngioVue from OptoVue. A Group of the patients were recruited to obtain further OCTA images on the Topcon device but we didn't add them to the study for several reasons will be listed below.Each commercially available OCTA device has its own algorithm that analyzes the date. For instance AngioVue uses the SSADA algorithm, whilst Zeiss AngioPlex uses OMAG algorithm. In his review on the commercially available OCTA devices. Turgut ${ }^{[24]}$ claimed that in a recent study, when algorithms included OMAG, speckle variance, phase variance, SSADA and correlation mapping were compared, it was found that OMAG, as the method utilisingcomplex OCT signals to contrast retinal blood flow provided the best visual result for the of retinal microvascular networks concerning image contrast and vessel connectivity ${ }^{[25]}$. The FDA approval was another issue to pay attention to Zeiss Medical Technology (Dublin, CA) received FDA clearance for its AngioPlex OCT angiography technology in April 2015, while AngioVue from Optovue got the approval for its analytics in software in June 2018, the OCTA system itself was approved in Feb 2016 ${ }^{[26]}$ Whereas other devices like Topcon Triton++ was only approved for Optical Coherence Tomography not OCT Angiography. Concerning quantification and data analysis, both the AngioPlex and AngioVue have the same capabilities, however after the AngioVue has received its FDA clearance it became superior. On the other, hand Triton ++ remainedless precedent in data quantification and analysis that why we didn't rely on data collected by it in our study. Other commercially available devices in Egypt like Heidelberg Spectralis OCTA remain very primitive when it comes to tissue 
Optical Coherence Tomography Angiography Study for Evaluation of Microvascular...

segmentation and quantification, it only gives a nice picture without any numerical date, the basic software interface isn't yet refined. Collected data: between 2015 and 2016, published studies on OCTA were relatively scarce, however in 2017 and 2018 published data has become fairly abundant, that an investigator can compare his findings to other's and also can refer to the normative data basis published by others on the issue of quantified data using OCTA so that a study on a given disease can be condcuted on OCTA without being in need of a control group.

Coscas ete.l ${ }^{[27]}$ published normative data for vascular density in superficial and deep capillary plexus of healthy adults assessed by optical coherence tomography angiography, they provided an age-related VD mapping data using OCT angiography in healthy subjects. They used the AngioAnalytics software by OptoVue to quantify vascular density and Foveal avascular zone. Part of the outcomes of such study were incomparable with our study, since it was doing an age-related map using a different prototype software, however the FAZ area mean value is consistent with our findings. Coscas et $a l .{ }^{[27]}$ postulate that the mean FAZ area in all age groups is $0.28+10 \mathrm{~mm}^{2}$. Our statistical analysis showed that the mean FAZ for the control group was $0.26+0.7 \mathrm{~mm}^{2}$ for our control group. Another study conducted by Mary et al. ${ }^{[28]}$ and evaluated the ability of measurements of retinal microvasculature using OCTA to distinguish healthy eyes from eyes with DR. The study was done to differentiate between retinal microvasculature of normal participants, and participants with type II diabetes. This study used the same device and software we have used in our study to examine 50 eyes of 26 participants with diabetes, and 50 eyes of 25 normal participants (Compared to our sample volume, where examined 40 eyes of 20 patients in each group). However the pivotal dissimilarities between this study and ours are the duration of diabetes and the stage of diabetic retinopathy for the diabetic group. The mean duration of diabetes for the mentioned study was 18.7 years whereas in our study it was $6.84+1.20$ years. In our study we have excluded any participant showing any frank manifestation of diabetic retinopathy, whereas in the mentioned study, participants with different stages of diabetic retinopathy were
included.Mary et $\boldsymbol{a l} .^{[28]}$ found that the mean perfusion density for the healthy group in the superficial retinal layers was SRL 0.419 , the mean vascular density was 22.5 , the mean FAZ area perimeter and circularity indexsequentially were 0.252 .05 and 0.82 . We got relatively close numbers, that we found the mean perfusion density in the SRL $0.36 \pm 0.06$ the mean VD in SRL 20.4 \pm 1.56 . Mean FAZ area perimemter and circularity index sequentially are $0.26 \pm 0.07$ and $2.18 \pm 0.33$ and $0.67 \pm 0.08$. For the diabetic group the mean PD in SRL was 0.409, mean VD in SRL was 21.2 and FAZ area, perimeter and circularity index sequentially are $0.26,2.32$ and 0.78 . In our study we had a PD in the SRL of $0.33 \pm 0.04$ VD in SRL of $17.75 \pm 2.91$ and FAZ area, perimeter and circularity index sequentially $0.31 \pm 0.12$, $2.46 \pm .58$ and $0.63 \pm 0.12$. Both studies found a statistically significant difference in all variables measured.For repeatability and reproducibility of SRL VD, PD and foveal vascular zone FAZ values measurement by using the AngioPlex OCTA device from CarlZeiss Meditec, Germany. Jianqinet al. ${ }^{[29]}$ published a study on that issue concluding that VD and PD of the superficial retinal vasculature can be obtained from OCTA images with high levels of repeatability and reproducibility but can vary with scan pattern and location. Each eye underwent 3 repeated scans with 3 instruments for a total of 9 acquisitions. Eyes were randomly assigned to scanning with a $3 \times 3-\mathrm{mm}$ or $6 \times 6-\mathrm{mm}$ pattern. Eyes were excluded from subsequent analysis if any acquisition had signal strength of less than 7 , she defined reproducibility as the agreement between devices of the same type, while repeatability was the agreement in measurements within a device.To avoid repeatability and reproducibility errors, we obtained each image four times successively. If there was a significant scan quality difference between images, we increased the number. We found no statistically significant difference between images taken with the same scan quality by the same investigator. The limitations of this study included the relatively small sample volume of both the diabetic group and the control group. There was no normative database available for the time being on ethnic differences in VD, PD and FAZ values so that we couldn't have selected our control volunteers on ethnic basis, we supposed that all had the same 
values as long as they belonged to the same age group. Fortunately, we didn't find an intergroup statistically significant difference in VD and PD and FAZ values, but these needs to be reassured by further studies with larger sample volumes and ethnic classification of the groups.

\section{References}

1. International Council of Ophthalmology (2017): Guidelines for diabetic eyecare. ICO.,http://www.icoph.org/downloads/ICOG uidelinesforDiabeticEyeCare.pdf.

2. Keech A, Simes RJ, Barter P et al.(2005): Effects of long-term fenofi brate therapy on cardiovascular events in 9795 people with type 2 diabetes mellitus (the FIELD study): randomized controlled trial. Lancet, 366:1849-1861.

3. Patel A, MacMahon S, Chalmers J et al. (2008): Intensive blood glucose control and vascular outcomes in patients with type 2 diabetes. N. Engl. J. Med., 358:2560-2572.

4. Mitchell $P$ and Wong TY (2008): DIRECT new treatments for diabetic retinopathy. Lancet, 372:1361-1363.

5. Wong TY, Liew G, Tapp RJ et al. (2008): Relation between fasting glucose and retinopathy for diagnosis of diabetes: three population-based cross-sectional studies. Lancet, 371:736-743.

6. Steinle NC, Ambati J and Darlene AD (2010): Retinal vasculopathies: diabetic retinopathy. In: Encyclopedia of the Eye. Academic Press, Oxford.

7. Parsons-Wingerter $\mathbf{P}$, Radhakrishnan $\mathbf{K}$, Vickerman MBet al.(2010): Oscillation of angiogenesis with vascular dropout in diabetic retinopathy by VESselGENeration analysis (VESGEN). Invest. Ophthalmol. Vis. Sci., 51:498-507.

8. Mendis KR, Balaratnasingam C, Yu P et al. (2010): Correlation of histologic and clinical images to determine the diagnostic value of fluorescein angiography for studying retinal capillary detail. Invest. Ophthalmol. Vis. Sci., 51:5864-5869

9. Cheng SC and Huang YM (2003): A novel approach to diagnose diabetes based on the fractal characteristics of retinal images. Trans. Inf. Technol. Biomed., 7:163-170
10. de Carlo TE, Chin AT, BoniniFilho MA et al. (2015): Detection of microvascular changes in eyes of patients with diabetes but not clinical diabetic retinopathy using optical coherence tomography angiography. Retina, $35: 2364-2370$

11. Matsunaga D, Yi J, Puliafito $C A$ and Kashani AH (2014): OCT angiography in healthy human subjects. Ophthalmic Surg. Lasers Imaging Retina, 45:510-515.

12. Yu J, Jiang $C$, Wang $X$ et al. (2015): Macular perfusion in healthy Chinese: an optical coherence tomography angiogram study. Invest .Ophthalmol. Vis. Sci., 56:32123217

13. de Carlo TE, Chin AT, BoniniFilho MA et al.(2015): Detection of microvascular changes in eyes of patients with diabetes but not clinical diabetic retinopathy using optical coherence tomography angiography. Retina, 35:2364-2370

14. Ishibazawa $A$, Nagaoka $T$, Takahashi A et al.(2015) : Optical coherence tomography angiography in diabetic retinopathy: a prospective pilot study. Am. J. Ophthalmol., 160:35-44.

15. Jia Y, Tan O, Tokayer J et al.(2012): Splitspectrum amplitudedecorrelation angiography with optical coherence tomography. Opt. Express, 20:4710-4725

16. Tokayer J, Jia Y, Dhalla AHet al (2013): Blood flow velocity quantification using splitspectrum amplitude-decorrelation angiography with optical coherence tomography. Biomed. Opt. Express,4:19091924

17. Agemy SA, Scripsema NK, Shah CM et al.(2015): Retinal vascular perfusion density mapping using optical coherence tomography angiography in normal and diabetic retinopathy patients. Retina, 35:2353-2363.

18. Mary K D, Lin A et al.(2017): Quantification of retinal microvascular density in optical coherence tomographic angiography images in diabetic retinopathy. JAMA Ophthalmol.,135(4):370-376.

19. Cheung N and Wong TY (2008): Diabetic retinopathy and systemic vascular complications. Prog. Retina Eye Res., 27:161-176. 
20. Tam J, Dhamdhere K, Tiruveedhula $P$ et al.(2012): Subclinical capillary changes in non proliferative diabetic retinopathy. Optom. Vis. Sci., 89(5):692-703.

21. Russmann C, Mansoor M and Amiji (2017) :Molecular imaging of subclinical diabetic retinopathy. J. Ophthalmic Vis. Res., 12(2): $129-131$

22. Frimmel S, Zandi S, Sun $\mathrm{D}$, Zhang $\mathrm{Z}$ et al.(2017): Molecular imaging of retinal endothelial injury in diabetic animals. J. Ophthalmic Vis. Res., 12(2): 175-182.

23. Hafezi-Moghadam Aet al. (2010): Superior sensitivity of novel molecular imaging probe: Simultaneously targeting two types of endothelial injury markers. FASEB J., 24:1532-1540.

24. Burak T (2016): Optical Coherence Tomography Angiography - A General View. European Ophthalmic Review, 10(1):39-42
25. Klein R, Klein BE, Moss SE, Davis MD and DeMets DL (1984): The Wisconsin epidemiologic study of diabetic retinopathy. II. Prevalence and risk of diabetic retinopathy when age at diagnosis is less than 30 years. Arch Ophthalmol., 102:520-528.

26. Zhang A, Zhang $Q$, Chen CL and Wang RK (2015): Methods and algorithms for optical coherence tomography-based angiography: areview and comparison.J. Biomed. Opt.,20:100901-100909.

27. Coscas F, Sellam A, Glacet- Bernard A et al. (2016): Normative data for vascular density in superficial and deep capillary plexuses of healthy adults assessed by optical coherence tomography angiography. Invest. Ophthalmol. Vis. Sci., 57:211-223.

28. Durbin M, An L, Shemonski N et al.( 2017) :Quantification of microvascular density in optical Coherence Tomography Angiography in diabetic retinopathy. AMA Ophthalmol., 135(4):370-376.

29. Lei J, Durbin M, Shi Y et al.(2017): Repeatability and reproducibility of superficial macular retinal vessel density measurements using Optical Coherence Tomography Angiography in face images.JAMA Ophthalmol.,135(10):10921098 